\title{
RASUQAM : le thésaurus de descripteurs de l'Université du Québec à Montréal (UQAM)
}

\section{RASUQAM: The Thesaurus of Descriptors of the Université du Québec à Montréal (UQAM) RASUQAM: El tesauro de descriptores de la Universidad de Québec en Montreal (UQAM)}

\section{Benoit Bilodeau}

Volume 52, numéro 2, avril-juin 2006

Les langages documentaires

URI : https://id.erudit.org/iderudit/1030014ar

DOI : https://doi.org/10.7202/1030014ar

Aller au sommaire du numéro

Éditeur(s)

Association pour l'avancement des sciences et des techniques de la documentation (ASTED)

ISSN

0315-2340 (imprimé)

2291-8949 (numérique)

Découvrir la revue

Citer cet article

Bilodeau, B. (2006). RASUQAM : le thésaurus de descripteurs de l'Université du Québec à Montréal (UQAM). Documentation et bibliothèques, 52(2), 109-120. https://doi.org/10.7202/1030014ar
Résumé de l'article

RASUQAM est un thésaurus encyclopédique développé depuis 1994 par le Service des bibliothèques de l'Université du Québec à Montréal. Conforme au format MARC 21, il compte aujourd'hui environ 40000 descripteurs, dont près de $70 \%$ sont des noms communs. Proche du langage couramment utilisé par les usagers, RASUQAM facilite la recherche et la navigation. Le présent article en retrace l'historique et décrit ses caractéristiques principales ainsi que sa structure, son état actuel et sa croissance. À l'aide d'exemples, il présente les différentes catégories de descripteurs qui s’y trouvent et fournit des précisions sur les zones et sous-zones MARC utilisées. Un parallèle est enfin établi avec le Répertoire des vedettes-matière (RVM) de l’Université Laval, langage documentaire réputé et très utilisé.
Tous droits réservés (c) Association pour l'avancement des sciences et des techniques de la documentation (ASTED), 2006
Ce document est protégé par la loi sur le droit d'auteur. L'utilisation des services d’Érudit (y compris la reproduction) est assujettie à sa politique d'utilisation que vous pouvez consulter en ligne. 


\author{
BENOIT BILODEAU \\ Service d'analyse documentaire \\ Bibliothèque, Université du Québec à Montréal \\ Bilodeau.benoit@uqam.ca
}

\begin{abstract}
RÉSUMÉ | ABSTRACTS | RESUMEN
RASUQAM est un thésaurus encyclopédique développé depuis 1994 par le Service des bibliothèques de l'Université du Québec à Montréal. Conforme au format MARC 21, il compte aujourd'hui environ 40000 descripteurs, dont près de $70 \%$ sont des noms communs. Proche du langage couramment utilisé par les usagers, RASUQAM facilite la recherche et la navigation. Le présent article en retrace l'historique et décrit ses caractéristiques principales ainsi que sa structure, son état actuel et sa croissance. $\grave{A}$ l'aide d'exemples, il présente les différentes catégories de descripteurs qui s'y trouvent et fournit des précisions sur les zones et sous-zones MARC utilisées. Un parallèle est enfin établi avec le Répertoire des vedettes-matière (RVM) de l'Université Laval, langage documentaire réputé et très utilisé.
\end{abstract}

\section{RASUQAM: The Thesaurus of Descriptors of the Université du Québec à Montréal (UQAM)}

RASUQAM is an encyclopaedic thesaurus developed in 1994 by the library service of the Université du Québec à Montréal. In compliance with MARC21, it has over 40,000 descriptors, of which nearly $70 \%$ are nouns. Similar to the language used by the users of the library, RASUQAM simplifies research and browsing. This article outlines the history of RASUQAM and describes its features, structure as well as its current status and growth. Using examples, the author describes the different categories of descriptors as well as the zones and sub-zones of MARC that are used. A comparison is established with the Répertoire de vedettes-matière de l'Université Laval (RVM), a widely used and recognised classification schedule.

\section{RASUQAM. El tesauro de descriptores de la Universidad de Québec en Montreal (UQAM)}

RASUQAM es un tesauro enciclopédico desarrollado desde 1994 por el Servicio de bibliotecas de la Universidad de Québec en Montreal. Conforme al formato MARC21, cuenta actualmente con aproximadamente 40 ooo descriptores, de los que casi un $70 \%$ son nombres comunes. Cercano al lenguaje comúnmente utilizado por los usuarios, RASUQAM facilita la búsqueda y la navegación. El presente artículo recuerda el historial y describe las características principales así como la estructura, el estado actual y el crecimiento de RASUQAM. Con la ayuda de ejemplos, presenta las diferentes categorías de los descriptores que ahi se encuentran y brinda precisiones sobre las zonas y sub zonas MARC utilizadas. Finalmente se establece un paralelo con el Repertorio de Encabezados de Materia de la Universidad Laval, lenguaje documental reputado y muy utilizado.
L A MOITIÉ DES RECHERCHES dans les catalogues de bibliothèque sont effectuées par sujet. C'est la nette préférence pour cette approche et le souci de mieux répondre aux besoins de ses usagers qui a incité le Service des bibliothèques de l'Université du Québec à Montréal (UQAM) à développer et à appliquer, depuis novembre 1994, son propre thésaurus de descripteurs. Construit à partir de la documentation indexée, le Répertoire des AutoritésSujet de l'UQAM (RASUQAM) est un thésaurus encyclopédique, conforme au MARC 21, qui compte aujourd'hui environ 40000 descripteurs.

L'examen des requêtes formulées par les usagers du catalogue BADADUQ (BAnque de Données à Accès Direct de l'Université $d u$ Québec ${ }^{1}$ ) révèle que ceux-ci utilisent spontanément le langage naturel ${ }^{2}$. Par ailleurs, le contexte qui prévalait au début du projet s'est considérablement transformé avec l'émergence et la croissance fulgurante d'Internet. Encore là, c'est la recherche par sujet et le langage naturel qui règnent pratiquement sans partage. La tendance à l'autonomie en matière de recherche d'information, qui se vérifiait déjà en $1992^{3}$, ne se dément certainement pas aujourd'hui. Les habitudes de recherche d'information développées avec l'utilisation généralisée des moteurs de recherche influencent à coup sûr l'interrogation du catalogue.

À l'heure où la recherche par sujet tend à conjuguer le catalogue de bibliothèque, les bases de données et Internet, ce sont là des éléments que les systèmes et les langages documentaires doivent prendre en considération afin de mieux aider l'usager dans sa quête d'information. Ces éléments confirment

1. Il est possible, au moment de la parution de cet article, que le nom «BADADUQ» ait été changé à la suite des démarches entreprises pour trouver un nouveau nom au catalogue de l'UQAM.

2. Un examen des requêtes par sujet adressées à BADADUQ, le 16 mars 2000, montre que les usagers interrogeaient très souvent avec des expressions en langage naturel (ex. Centre de la petite enfance; Stratégie pédagogique; Père de famille monoparentale, etc.) et faisaient très peu de cas des unitermes (voir note $\mathrm{n}^{\circ}$ 7) qui étaient alors le mode de repérage offert par sujet. Un échantillon des requêtes du 7 février 2004 révèle la même approche du catalogue par les usagers.

3. L'enquête auprès des usagers de BADADUQ montre aussi qu'en 1992, déjà, la moitié des usagers de l'UQAM apprenaient à utiliser BADADUQ par eux-mêmes. (Enquête auprès des usagers de BADADUQ: Rapport final, ibid., p. A2-2.) 
aussi la justesse du choix d'un langage documentaire à base de descripteurs qui, en plus de répondre aux attentes de nos usagers, peut également profiter à ceux des autres institutions qui décident de conserver, dans les notices bibliographiques de leur catalogue, les descripteurs trouvés dans les notices dérivées de l'UQAM ${ }^{4}$.

Cet article fait d'abord l'historique du projet. Suivent la présentation des différentes caractéristiques de RASUQAM et la description de létat et de la croissance du thésaurus, dans les deuxième et troisième parties. Les différentes catégories de descripteurs sont introduites dans la quatrième partie et les modalités d'utilisation du format MARC font l'objet de la cinquième partie. Enfin, nous établissons quelques points de comparaison entre descripteurs et vedettesmatière.

\section{Historique du projet}

Utilisés à partir de 1972 , les unitermes ${ }^{5}$ ont été le premier langage d'indexation de BADADUQ. Peu après, les difficultés inhérentes à ce langage documentaire ont fait surface: silence et bruit ${ }^{6}$, absence de contrôle du vocabulaire, aide inexistante, etc.

Dans un rapport remis en 1978, Suzanne BertrandGastaldy, qui travaillait à cette époque au Service de l'analyse documentaire de l'UQAM, critique sévèrement l'utilisation des unitermes:

"Nous avons pu constater à quel point il est gênant de faire l'indexation sur les mots plutôt que sur les concepts. La méthode des unitermes mise au point par Mortimer Taube est désuète; elle crée beaucoup de fausses combinaisons et empêche d'établir des relations

4. Cela se vérifie déjà dans le cadre du projet québécois de traitement partagé de certaines collections de documents électroniques (projet TRAP parrainé par la CREPUQ), par lequel l'UQAM fournit des notices bibliographiques aux autres institutions participantes.

5. Résultant du processus de décomposition des concepts, les unitermes sont des mots sur lesquels est appliquée la règle du masculin/singulier. Cette règle prescrit que, sauf exception, les substantifs sont au singulier et les qualificatifs au masculin singulier.

Exemples : Concepts Unitermes correspondants 1. Base de données BASE + DONNEE

2. Maladie de la vache folle MALADIE + VACHE + FOU

3. Sciences infirmières SCIENCE + INFIRMIER

L'application stricte de cette règle mène parfois au ridicule alors que l'eau salée devient de l'EAU SALE et que la femme violentée devient une FEMME VIOLENT!

6. Difficiles à maîtriser par les usagers, les unitermes génèrent à la fois du silence et du bruit au repérage. D'une part, le silence est dû au fait que les usagers n'appliquent généralement pas la règle du masculin-singulier afin de retrouver les documents qui traitent de leur sujet (sans compter les variantes que l'on peut aussi observer dans l'application de cette règle par les indexeurs eux-mêmes). D'autre part, le bruit est engendré par le grand nombre de notices obtenues, soit par de fausses combinaisons de mots, soit par la présence du mot recherché dans de nombreux concepts différents (par exemple, l'uniterme LINGUISTIQUE repêchera aussi bien les notices traitant de la Linguistique que celles traitant des Politiques linguistiques, des Minorités linguistiques, de l'Analyse linguistique, de la Géographie linguistique, etc.). entre concepts utiles pour guider l'utilisateur

dans sa recherche?.»

Les travaux menés dans les années suivantes par un groupe de travail interne (GTI - Groupe de travail sur l'indexation) concluent que la recherche par sujet est déficiente dans BADADUQ, n'étant ni conviviale ni intuitive, et que seul l'usager expérimenté ou celui dont la requête répond par bonheur à la règle du masculin-singulier des unitermes peut espérer en retirer quelque chose. Il est alors démontré qu'une recherche en unitermes n'obtient qu'un taux de pertinence de $25 \%$, alors que, pour une même recherche effectuée avec des descripteurs, ce taux peut atteindre $68 \%$. Les principales conclusions des études du GTI confirment celles du rapport Gastaldy:

a) les unitermes sont une des principales sources de bruit au repérage;

b) l'éclatement des concepts en unitermes crée un langage artificiel difficilement conciliable avec la démarche intellectuelle tant de l'usager que de l'indexeur;

c) les unitermes ne permettent ni contrôle ni gestion du vocabulaire, rendant le repérage plus laborieux;

d) les unitermes rendent impossible toute assistance terminologique pour l'usager et pour l'indexeur.

Le consensus s'établit au Service des bibliothèques de l'UQAM, avec l'appui du Comité des usagers des bibliothèques, sur la recommandation principale du GTI voulant que les concepts remplacent les mots comme base du futur langage d'indexation. Les descripteurs s'imposent comme solution aux problèmes générés par l'emploi des unitermes. Les vedettes-matière de l'Université Laval ne sont pas retenues à cause, d'une part, de la difficulté appréhendée d'utilisation et d'interprétation découlant de leur syntaxe contraignante et, d'autre part, de la volonté de demeurer proche du langage des bases de données et des thésaurus, ainsi que du langage utilisé par les usagers.

Par ailleurs, tout en effectuant leur travail quotidien d'indexation de la documentation, plusieurs des membres du Service de l'analyse documentaire ont travaillé au développement des différents aspects du système d'indexation qui allait être mis en place: rédaction des devis fonctionnels, liste des domaines d'emploi, stratégie de cohabitation entre les unitermes et les descripteurs, repérage et navigation (en collaboration avec des membres du personnel des bibliothèques), etc.

L'indexation par descripteurs est implantée de façon progressive à partir du 21 novembre 1994. À cette époque, l'importance n'est accordée ni au contrôle du

\footnotetext{
7. Suzanne Gastaldy, L'indexation dans Badaduq et ses répercussions sur le repérage. Montréal: UQAM, 1978, IV-7.
} 
vocabulaire, faute de politiques, ni au développement des relations sémantiques, faute d'un nombre suffisant de termes dans le thésaurus. Les descripteurs sont créés de façon relativement libre, héritage de plus de 22 ans d'indexation en unitermes.

L'adoption, en 1996, de politiques d'indexation et de gestion du vocabulaire ${ }^{8}$ amorce une opération de redressement et de consolidation du thésaurus, qui contient alors près de 15000 termes. L'année suivante, un mécanisme de vérification est instauré afin de garantir que les descripteurs soient créés dans le respect de ces politiques. Chaque nouveau terme doit désormais être validé par le gestionnaire du thésaurus avant d'être reconnu comme descripteur'.

En 1998, l'appellation «RASUQAM» est reconnue et intégrée à la liste des Codes de relations, de sources et de conventions de description MARC, autorisant ainsi linscription éventuelle de ses descripteurs dans les zones $6 \mathrm{XX}$ de la notice bibliographique ${ }^{10}$.

Comme tous les fichiers d'autorité du système documentaire SIGIRD (Système Intégré de Gestion Informatisée des Ressources Documentaires) utilisé à l'UQAM, c'est dans un format de données «maison" que fut d'abord développé RASUQAM. Les données d'autorité et bibliographiques ont dû être converties au format MARC à l'occasion du passage au système MANITOU, système documentaire développé pour remplacer SIGIRD. Nos descripteurs sont aujourd'hui entièrement compatibles avec le format MARC et se retrouvent dans les champs $6 \mathrm{XX}$ des notices bibliographiques.

Depuis juin 2002, date de l'implantation du module de catalogage de MANITOU, les descripteurs sont repérables et facilement utilisables pour la navigation thématique au catalogue.

RASUQAM, qui compte aujourd'hui environ 40000 descripteurs, est également utilisé pour indexer la bibliothèque numérique du centre de documentation du ministère de l'Éducation, du Loisir et du Sport (MELS) du Québec. Ce centre conserve et diffuse toutes les publications officielles imprimées, électroniques et audiovisuelles produites par le Ministère et les organismes relevant du ministre. De plus, l'utilisation de RASUQAM pour la création des métadonnées du site Web ministériel est présentement à l'étude.

8. Ces politiques, élaborées conjointement par le Service de l'analyse documentaire et des représentants des bibliothèques de l'UQAM, peuvent être consultées sur la page Web du Service de l'analyse documentaire de l'UQAM à l'adresse $h t t p: / /$ www.bibliotheques.uqam.ca/bibliotheques/serv_techniques/analyse/index.html.

9. Même sans avoir été validés, les candidats-descripteurs sont, dès leur création, utilisables pour l'indexation et de recherche.

10. Le code «rasuqam», inscrit en sous-zone $\$ 2$ des zones $6 \mathrm{XX}$, identifie les descripteurs de l'UQAM dans les notices bibliographiques. Ce code peut être utilisé par les bibliothèques qui importent nos notices ou par les institutions qui veulent utiliser notre langage documentaire.

\section{Caractéristiques de RASUQAM}

Puisqu'il respecte les principes directeurs pour l'établissement et le développement des thésaurus - AFNOR et ISO $2788^{11}-$, RASUQAM possède les caractéristiques communes à la plupart de ces outils. Ainsi, les descripteurs sont généralement au singulier. Chacun représente un concept et possède sa propre fiche d'autorité où sont inscrits ses synonymes (EP) et établit ses relations hiérarchiques (TG et TS) et associatives (TA) avec d'autres termes présents dans le thésaurus. Il fait appel à la post-coordination des descripteurs lors du repérage pour retrouver les sujets composés de plusieurs concepts.

Par ailleurs, RASUQAM présente des particularités qui en font un thésaurus unique:

$\triangleright A$ posteriori: construit progressivement, par extraction des termes des documents à indexer (approche analytique plutôt que synthétique).

$\triangleright$ Évolutif et ouvert: tout nouveau terme pertinent est rapidement intégré au thésaurus.

$\triangleright$ Encyclopédique: construit à partir de nos collections multidisciplinaires.

$\triangleright$ Domaines d'emploi: le domaine d'emploi ${ }^{12}$ attribué à chaque descripteur est un critère utilisable pour raffiner la stratégie de recherche ou pour connaitre la liste de tous les descripteurs d'un champ particulier.

$\triangleright$ Définition: la majorité des descripteurs s'accompagnent d'une définition ou d'un contexte d'utilisation.

$\triangleright$ Bilingue: les équivalents anglais sont ajoutés comme synonymes aux descripteurs ${ }^{13}$; cela facilite le repérage ainsi que l'indexation en français des documents de langue anglaise.

$\triangle$ MARC 21: format utilisé comme structure de données pour les descripteurs.

$\triangleright$ Catégories de descripteurs: outre les noms communs, on y trouve aussi des noms d'organismes, des titres-sujet, des noms de lieux, des termes relatifs à la chronologie, des descripteurs de forme ainsi que quelques descripteurs non contrôlés.

$\triangleright$ Liaison RVM: la correspondance avec les vedettesmatière du RVM est établie, dans un grand nombre de cas, à l'aide d'une zone de liaison.

11. Association française de normalisation (AFNOR), Règles d'établissement des thésaurus monolingues, 1981, NF Z47-100 et Organisation internationale de normalisation (ISO), Documentation - Principes directeurs pour létablissement et le développement de thésaurus monolingues, 1986, ISO 2788 (pour les relations sémantiques surtout). Nous respectons aussi, généralement, les Règles de catalogage anglo-américaines (RCAA2) relatives aux vedettes de collectivités et aux titres-sujet (nom/titre et titre uniforme).

12. La liste des 49 domaines d'emploi est disponible sur la page Web du Service de l'analyse documentaire.

13. Le bilinguisme se limite, toutefois, à la présence des formes rejetées. Il n'y a pas de forme acceptée en anglais et RASUQAM ne peut pas servir à l'indexation en anglais. Les multiples formes anglaises ne servent qu'au repérage. 
Tableau 1: Catégories de descripteurs dans RASUQAM

\begin{tabular}{lrc}
\hline Noms communs & 26375 & $68,3 \%$ \\
\hline Collectivités & 4741 & $12,3 \%$ \\
\hline Titres-sujet & 2814 & $7,3 \%$ \\
\hline Toponymes & 3975 & $10,3 \%$ \\
\hline Chronologie & 273 & $0,7 \%$ \\
\hline Non contrôlés & 339 & $0,9 \%$ \\
\hline Descripteurs de forme & 96 & $0,2 \%$ \\
\hline TOTAL & 38613 & $100 \%$ \\
\hline
\end{tabular}

Tableau 2: Nombre de descripteurs dans quelques thésaurus couramment utilisés

\begin{tabular}{lll}
\hline UNESCO* $^{*}$ & 4261 & Thésaurus général de l'UNESCO \\
\hline OCDE $^{*}$ & 5272 & Macrothésaurus de l'OCDE \\
\hline EUROVOC* & 6645 & $\begin{array}{l}\text { Thésaurus des institutions de l'Union } \\
\text { européenne }\end{array}$ \\
\hline AGROVOC* & 16700 & Thésaurus de la FAO \\
\hline MeSH* & 23880 & Medical Subject Headings \\
\hline RASUQAM & 26375 & Thésaurus de l'UQAM \\
\hline RAMEAU & 88462 & $\begin{array}{l}\text { Répertoire d'autorités-matière } \\
\text { encyclopédique de la BNF }\end{array}$ \\
\hline RVM & 157200 & $\begin{array}{l}\text { Répertoire de vedettes-matière de } \\
\text { l'Université Laval }\end{array}$ \\
\hline
\end{tabular}

* Les données pour ces thésaurus incluent des noms géographiques et des noms d'organismes. Les chiffres des autres thésaurus ne tiennent compte que des noms communs.

Le thésaurus ne contient pas de formes inversées ni de subdivisions telles qu'on les trouve dans les vocabulaires normalisés de type vedettes-matière. Il se rapproche ainsi du langage des bases de données et du langage usuel de recherche des usagers. Cette approche peut rendre le thésaurus facilement utilisable pour lancer une recherche sur Internet au moyen des moteurs disponibles ${ }^{14}$.

\section{État et croissance de RASUQAM}

Au 31 mai 2005, RASUQAM comptait 38613 descripteurs et 62175 renvois (ou non-descripteurs). Si la grande majorité des termes (26375) sont des noms communs, on y rencontre aussi des noms d'organismes, des titres d'œuvres, des noms géographiques, des termes relatifs à la chronologie, des descripteurs de forme ainsi que des descripteurs non contrôlés. (Voir dans le tableau 1 les statistiques par catégories de descripteurs.)

14. C'est ce que proposent, par exemple, le Thésaurus canadien d'alphabétisation (thesaurusalpha.org) ou le thésaurus de la Banque mondiale qui permettent, à partir de chaque terme (formes acceptées et rejetées), d'interroger Internet avec Yahoo! et Google.
Ce sont les indexeurs du Service de l'analyse documentaire qui procèdent à la création des descripteurs nécessaires à l'indexation des collections. Chacun s'occupe de la terminologie et des réseaux sémantiques dans les disciplines dont il a la responsabilité. Le contrôle et l'homogénéité de l'ensemble sont assurés par le gestionnaire du thésaurus.

RASUQAM se compare aujourd'hui avantageusement aux thésaurus et répertoires de vedettes-matière les plus courants ${ }^{15}$, comme en témoigne le tableau 2.

\section{Croissance de RASUQAM}

Depuis ses débuts en novembre 1994, jusqu'en mai 2005 , le thésaurus a été augmenté en moyenne de 306 descripteurs par mois. Cette moyenne s'établit à 215 pour la période 2000-2005, alors qu'elle n'est plus que de $\mathbf{1 4 5}$ pour la dernière année. Si l'on ne considère que les noms communs, les chiffres pour ces trois périodes sont ramenés respectivement à 209 , 131 et 81 descripteurs par mois (voir tableau 3). S'il fallait au début créer tous les descripteurs requis pour chacun des documents à indexer, il n'y a plus aujourd'hui que $8 \%$ des documents indexés ${ }^{16}$ qui nécessitent la création d'un nouveau descripteur.

\section{Coordination}

Comme tous les thésaurus, RASUQAM est un langage documentaire destiné à l'utilisation en contexte de post-coordination. C'est donc à l'usager, qui compose sa requête, que revient la tâche et la liberté d'assembler, en fonction de sa stratégie de recherche, les descripteurs qui ont été attribués séparément aux documents au moment de l'indexation.

L'extension conceptuelle - ou couverture thématique - d'un thésaurus détermine le degré de précoordination conceptuelle et terminologique nécessaire. Dans un thésaurus spécialisé où le caractère polysémique du descripteur est déjà circonscrit par son domaine d'application, la présence de termes

15. Source: Données publiées dans Internet par les différents services documentaires.

16. Le Service de l'analyse documentaire indexe en moyenne 22000 documents par année. 
Tableau 3: Nombre moyen de descripteurs créés sur une base mensuelle

\begin{tabular}{l|c|c|c|c|c|c|c|c}
\hline \multirow{2}{*}{ Période } & \multicolumn{2}{|c|}{ RASUQAM } & \multicolumn{3}{c|}{ Noms communs } & \multicolumn{3}{c}{ Autres catégories } \\
\cline { 2 - 11 } & total & mensuel & total & $\%$ & mensuel & total & $\%$ & mensuel \\
\hline $1994-2005$ & 38613 & 306 & 26375 & $68 \%$ & 209 & 12238 & $32 \%$ & 97 \\
\hline $2000-2005$ & 12928 & 215 & 7889 & $61 \%$ & 131 & 5028 & $39 \%$ & 84 \\
\hline $2004-2005$ & 1742 & 145 & 976 & $56 \%$ & 81 & 766 & $44 \%$ & 64 \\
\hline
\end{tabular}

Tableau 4: Ratio d'équivalence dans RASUQAM

\begin{tabular}{lc}
\hline EP & Ratio d'équivalence \\
\hline Tous & 2,0 \\
\hline français & 1,2 \\
\hline anglais & 0,8 \\
\hline
\end{tabular}

généraux sera moins problématique que dans un thésaurus encyclopédique ${ }^{17}$. Dans ce dernier cas, une certaine pré-coordination favorisant la précision de l'indexation et de la recherche sera nécessaire afin de réduire l'ambiguïté des termes.

En l'absence de termes appropriés dans RASUQAM, l'indexeur a le choix d'utiliser plusieurs descripteurs existants ou de créer un nouveau descripteur plus spécifique. Les règles et les critères de sélection énoncés dans les politiques de gestion du vocabulaire encadrent le développement du thésaurus et aident à décider de l'utilisation éventuelle d'une expression pré-coordonnée ${ }^{18}$. La pré-coordination peut dépasser le terme composé monoconceptuel et introduire plus d'un concept dans une même expression (ex.: Conservation des ceuvres d'art). Les termes ainsi obtenus représentent mieux les concepts utilisés dans la littérature et permettent aussi de construire des réseaux sémantiques plus étoffés.

Le ratio de pré-coordination correspond au nombre de mots significatifs contenus dans les descripteurs (par exemple, Conservation des ressources naturelles contient trois mots significatifs). Une mesure effectuée il y a trois ans sur un échantillon aléatoire de descripteurs de noms communs indique un ratio de 1,9. Un second échantillon, couvrant la période 20002005 , donne un ratio de 2,0. Ces résultats respectent le ratio recommandé ${ }^{19}$ qui se situe entre 1,5 et 2 .

17. Par exemple, le terme "Conservation» trouve plus facilement sa place dans un thésaurus en environnement ou en art que dans un thésaurus encyclopédique où il indexerait des documents aux sujets très variés qu'il serait nécessaire de filtrer en rajoutant d'autres termes à la requête.

18. En plus du document à indexer, de nombreuses sources sont consultées pour la création des descripteurs: banques terminologiques (GDT et Termium), thésaurus, dictionnaires, encyclopédies, répertoires de vedettes-matière, etc. Internet est aussi une mine d'information largement utilisée. À l'occasion, des spécialistes peuvent également être consultés.

19. Michèle Hudon, Le thésaurus: Conception, élaboration, gestion. Montréal: ASTED, 1996, p. 96.

\section{Synonymie}

Dans RASUQAM, le contrôle de la synonymie se fait par l'établissement de relations d'équivalence avec les termes français et les termes anglais. Adjoints comme formes rejetées (EP) aux descripteurs, les synonymes assurent un repérage plus exhaustif et une indexation plus cohérente.

Exemple : Librairie en ligne

EP Cyberlibrairie

Librairie électronique

Librairie virtuelle

Librairie sur Internet

eBookstores

Electronic bookstores

Internet bookstores

Online bookstores

Virtual bookstores

Web bookstores

Dans cet exemple, l'indexation sera toujours faite avec «Librairie en ligne», alors que le repérage avec n'importe lequel de ces 11 termes permettra à l'usager d'obtenir directement toutes les notices indexées ${ }^{20}$.

Le ratio d'équivalence, rapport entre le nombre de termes rejetés et le nombre de descripteurs contenus dans la banque, permet d'évaluer la richesse lexicale du thésaurus. Le tableau 4 fait état du ratio d'équivalence calculé pour l'ensemble de RASUQAM.

\section{Réseau sémantique}

La structure sémantique de RASUQAM comprend les relations hiérarchiques (termes génériques, TG, et termes spécifiques, TS) et les relations associatives (termes associés, TA). Ces relations permettent la navigation en situant le descripteur dans son environnement conceptuel. Elles permettent à l'usager de cerner plus facilement son sujet en lui offrant la possibilité de consulter et d'exploiter le thésaurus de façon logique.

RASUQAM est élaboré en fonction des documents à indexer et des besoins rencontrés. Pour cette raison, dans les premières années du projet, les descripteurs étaient forcément créés en dehors de

20. Dans le système MANITOU, contrairement à la plupart des SIGB, les formes acceptées et les formes rejetées entrent dans la constitution des index servant au repérage. 
Tableau 5: Nombre de titres indexés

\begin{tabular}{llccc}
\hline Période & Langage & Titres indexés & Indexeurs & Moyenne \\
\hline $1993-1994$ & Unitermes & 24018 & 9 & 2669 \\
\hline $2000-2001$ & Descripteurs & 22694 & 7 & 3242 \\
\hline
\end{tabular}

tout réseau sémantique, puisque les termes auxquels ils auraient dû être rattachés n'existaient pas encore. Maintenant que le thésaurus a atteint une taille respectable, aucun descripteur n'est créé orphelin (c'est-àdire sans relation sémantique), puisqu'il se trouve presque toujours quelques termes auxquels le relier. Lorsque ces termes n'existent pas, ils sont créés pour compléter la structure sémantique. Nous en profitons souvent pour développer un réseau de liens à partir de descripteurs qui, à l'origine, avaient été créés orphelins ou avec un réseau rudimentaire.

L'établissement des relations sémantiques, tel qu'appliqué dans RASUQAM, répond à un certain nombre de critères qui se trouvent, pour la plupart, dans les normes internationales (ISO 2788, AFNOR, etc.) ou dans des guides pratiques sur la construction de thésaurus (Aitchison et Gilchrist, 1992; Hudon, 1994, etc.). Nous appliquons aussi quelques règles «maison» adaptées aux collectivités-sujet et aux titressujet.

Au 31 mai 2005, le ratio de connectivité, c'est-àdire le nombre moyen de liens TG, TS et TA entre les descripteurs du thésaurus, s'établissait à 2,9. Ce ratio passe à 3,3 pour les descripteurs validés. Les noms communs étaient interconnectés par un total de 77150 relations sémantiques.

\section{Mise à jour}

Le processus de mise à jour s'intègre au développement quotidien de RASUQAM. Les ajouts et modifications, effectués directement dans la base de données, deviennent aussitôt disponibles pour tous les indexeurs.

La mise à jour, qui consiste souvent à combler des lacunes conceptuelles et terminologiques, vise à répondre aux besoins de l'indexation et à améliorer la qualité des dossiers existants et de l'ensemble du thésaurus. Les cas suivants sont rencontrés:

$\triangleright$ Création de nouveaux descripteurs.

Exemples: 1. Généalogie génétique

2. Internet des objets

3. Robot de loisir

$\triangleright$ Création d'un descripteur plus spécifique pour éviter l'utilisation de plusieurs descripteurs généraux.
Exemples : 1. Psychologie de l'adolescent

en remplacement de

1. Psychologie

2. Adolescent

$\triangleright$ Transformation d'une forme rejetée en descripteur.

Exemple : 1. Conciliation travail-vie personnelle

EP Conciliation travail-famille

devenu deux descripteurs distincts au thésaurus :

1. Conciliation travail-vie personnelle

2. Conciliation travail-famille

$\triangleright$ Ajout de nouveaux synonymes.

Exemple: 1. Partenariat public-privé

EP PPP

$\triangleright$ Inversion d'une forme acceptée et d'une forme rejetée.

Exemple: 1. Politique extérieure

EP Politique étrangère

devenu

1. Politique étrangère

EP Politique extérieure

$\triangleright$ Enrichissement du réseau sémantique d'un descripteur.

$\triangleright$ Ajout de définitions, sources, liaisons avec le RVM, etc.

$\triangleright$ Correction d'erreurs typographiques.

\section{Productivitée1}

Un changement de système d'indexation ne peut se faire sans conséquences notables sur les activités du service impliqué. Le tableau 5 compare la moyenne de titres indexés par indexeur en 1993-1994 (dernière année complète d'indexation avec les unitermes), avec celle de l'année 2000-2001, dernière année complète d'indexation en descripteurs avant l'implantation du système MANITOU ${ }^{22}$.

21. Pour plus de détails, on pourra consulter Le système d'indexation de l'UQAM: État d'avancement. Disponible sur le site Web du Service de l'analyse documentaire de l'UQAM à l'adresse <http://www.bibliotheques.uqam.ca/bibliotheques/ serv_techniques/analyse/rasuqam/Rasuqam \%202002.doc>.

22. La comparaison se limite à la période SIGIRD pour ne pas introduire d'éléments relevant des systèmes informatiques différents utilisés depuis l'adoption des descripteurs (SIGIRD, puis MANITOU à partir de juin 2002) et qui auraient pu fausser les résultats. Il faut également considérer que l'équipe des indexeurs était sensiblement composée des mêmes personnes. 
Ces chiffres montrent que la productivité avec les descripteurs s'est accrue de $21 \%$ par rapport aux unitermes, et cela en dépit du plus grand nombre d'indexeurs qui travaillaient avec les unitermes (9) qu'avec les descripteurs (7). Michèle Hudon explique ainsi ce résultat:

«Cette hausse de productivité dans une équipe réduite et fortement sollicitée par un bon nombre d'autres tâches s'explique par la disponibilité de meilleurs outils de contrôle qui facilitent pour les indexeurs la prise de décision et les validations et évitent les retours sur du travail déjà accompli pour effectuer des corrections, normaliser des formes, etc. $^{23}$ "

Nous pouvons donc considérer qu'en dépit d'un contexte peu favorable marqué par la réduction du personnel à la suite d'importantes compressions budgétaires, dans les années 1990, l'élaboration du thésaurus et le changement de système d'indexation ont eu un effet positif sur le travail quotidien.

\section{Indexation avec RASUQAM}

Contrairement à la plupart des institutions d'enseignement, les indexeurs de l'UQAM ne peuvent pas utiliser tel quel le travail d'analyse effectué ailleurs. Les termes d'indexation trouvés dans les notices bibliographiques dérivées sont donc considérés ici au même titre que les autres éléments servant au travail d'analyse (table des matières, résumé, etc.).

Cette situation particulière procure une relative indépendance vis-à-vis des sources de catalogage. Ainsi, les termes d'indexation trouvés dans les notices des autres catalogues seront, s'il y a lieu, traduits en descripteurs et ajustés à nos politiques. L'analyse sera souvent effectuée plus en profondeur et exprimée avec des termes plus spécifiques.

Exemple: Quel rôle pour les chemins de fer en Europe de l'Est. Paris : OCDE, 2000.

Indexation dérivée :

1. Chemins de fer - Europe de l'Est

Indexation UQAM :

1. Chemins de fer

2. Développement économique

3. Europe de l'Est

4. Industrie ferroviaire

5. Politique des transports

6. Réforme administrative

7. Transport ferroviaire

23. Michèle Hudon, Évaluation du système d'indexation en descripteurs de la bibliothèque de l'Université du Québec à Montréal (UQAM), 2002. Disponible sur le site Web du Service de l'analyse documentaire de l'UQAM à l'adresse suivante: <http://www.bibliotheques.uqam.ca/bibliotheques/serv_techniques/analyse/ rasuqam/HUDONrasuqam.doc>.

\section{Catégories de descripteurs}

À l'exception des noms de personnes, toutes les catégories de descripteurs sont présentes dans RASUQAM: noms communs, noms d'organismes, titres d'œuvre, noms géographiques, chronologies et descripteurs de forme. Il y a même quelques descripteurs non contrôlés!

\section{Noms communs}

Cette catégorie de descripteur représente près de $70 \%$ de l'ensemble du thésaurus. Elle se compose de représentations d'entités concrètes (objets, matériaux, etc.) ou abstraites (actions, émotions, relations, qualités, propriétés, sciences, etc.).
Exemples : 1. Chien
2. Développement durable
3. Philosophie de l'histoire
4. Vent solaire

De façon générale, ces termes sont en français et au singulier. Le pluriel est cependant utilisé lorsqu'il est consacré par l'usage ou si le terme n'existe pas au singulier.

Exemples : 1. Arts martiaux

2. Relations internationales

3. Travaux publics

Les formes anglaises sont ajoutées comme formes rejetées afin de faciliter le repérage.

Exemple: 1. Agrotourisme
EP Farm holiday
Farm tourism
Farmhouse holiday

Les termes empruntés à d'autres langues sont utilisés comme descripteurs lorsqu'il n'existe pas d'équivalent en français ou si ces termes sont largement utilisés tels quels.
Exemples : 1. Bat mitsva
2. Lock-out
3. Ut pictura poesis

Les formes françaises peu usitées sont ajoutées comme formes rejetées.

$\begin{array}{ll}\text { Exemples : } & \text { 1. Arte povera } \\ & \text { EP Art pauvre } \\ & \text { 2. Marketing } \\ \text { EP Mercatique }\end{array}$

\section{Collectivités-sujet}

Cette catégorie de descripteurs comprend les noms d'organismes, d'institutions et d'associations. S'y retrouvent également les noms de réunions (événements sportifs, expositions, colloques, etc.), de navires, d'engins spatiaux, etc. Elle représente $12 \%$ de l'ensemble des descripteurs. 
Exemples : 1. Agence internationale de l'énergie atomique

2. Colisée de Rome

3. Festival international de jazz de Montréal

4. Titanic (Navire)

Les directives des RCAA2 sont généralement respectées pour l'établissement de descripteurs représentant des collectivités-sujet. Toutefois, RASUQAM n'hésite pas au besoin à s'éloigner des fichiers d'autorités nationaux pour adopter une forme naturelle, plus conviviale et plus proche des utilisateurs. C'est le cas, en particulier, lorsque le nom de la collectivité est dans une langue autre que le français ou l'anglais. La forme acceptée est alors établie en français lorsqu'une telle forme est couramment utilisée dans les ouvrages francophones.

$$
\begin{array}{ll}
\text { Exemples : } & \text { 1. Ballet du Kirov } \\
& \text { plutôt que: } \\
& \text { Leningradskii gosudarstvennyi } \\
\text { akademicheskii teatr opery } \mathrm{i} & \text { baleta imeni } \\
& \text { S.M. Kirova } \\
\text { 2. Parti communiste chinois } & \text { plutôt que: } \\
& \text { Zhongguo gong chan dang }
\end{array}
$$

\section{Titres-sujet}

Cette catégorie, qui représente $7 \%$ de l'ensemble des descripteurs dans RASUQAM, comprend les titres d'œuvres littéraires, artistiques, musicales ou autres qui font l'objet d'une critique ou d'une analyse.

Exemples: 1. Ancien Testament

2. Flaubert, Gustave, 1821-1880. Dictionnaire des idées reçues

3. Fauré, Gabriel, 1845-1924. Requiem

4. Québec (Province). Loi sur les normes du travail

L'article initial est conservé en début de titre, afin de respecter le nom choisi par l'auteur ou consacré dans les ouvrages de référence en langue française.

$$
\begin{array}{ll}
\text { Exemples : } & \text { 1. de Vinci, Léonard, 1452-1519. } \\
& \text { La Joconde } \\
& \text { 2. Vian, Boris, 1920-1959. Lécume } \\
\text { des jours }
\end{array}
$$

La forme du descripteur est établie en s'inspirant du chapitre 25 des RCAA2 et des spécifications du format MARC 21. Toutefois, quelques variantes sont apportées dans le but de présenter à l'usager une forme plus conviviale. C'est notamment le cas pour les traités, déclarations et protocoles à caractère international.
Exemples: 1. Déclaration universelle des droits de l'homme
plutôt que :
Nations Unies. Assemblée générale. Déclaration universelle des droits de l'homme
2. Protocole de Kyoto plutôt que:
Convention-cadre des Nations Unies sur les changements clima- tiques (1992).
Protocoles, etc., 1997 déc. 11

Pour les œuvres littéraires (à l'exception des classiques grecs) et les œuvres musicales, la forme acceptée est établie au titre original dans la langue de l'auteur. Les renvois nécessaires sont établis à partir des formes les plus courantes en français.
Exemples :
1. Bach, Johann Sebastian, 1685- 1750. Brandenburgische Konzerte
EP Bach, Johann Sebastian, 1685- 1750. Concertos brandebour- geois
2. César, Jules. De bello gallico
EP César, Jules. La guerre des Gaules
3. Shakespeare, William, 1564- 1616. Much ado about nothing
EP Shakespeare, William, 1564- 1616. Beaucoup de bruit pour rien

\section{Noms géographiques}

Cette catégorie de descripteurs comprend les noms de lieux géographiques naturels et artificiels, les noms d'entités administratives (pays, villes, etc.), ainsi que les noms de voies de communication. Elle compte pour $10 \%$ de l'ensemble des descripteurs du thésaurus.

$$
\begin{array}{ll}
\text { Exemples: } & \text { 1. Autoroute 1o (Québec) } \\
& \text { 2. Djakarta (Indonésie) } \\
\text { 3. Madagascar } \\
\text { 4. Seigneurie de Kamouraska } \\
\text { (Québec) } \\
\text { 5. Vallée du Nil }
\end{array}
$$

La forme directe du nom de lieu est généralement préférée à sa forme inversée.

$$
\begin{array}{ll}
\text { Exemples : } & \text { 1. Lac Témiscouata (Québec) } \\
& \text { plutôt que : } \\
& \text { Témiscouata, Lac (Québec) } \\
\text { 2. Montagne Pelée (Martinique) } & \text { plutôt que: } \\
& \text { Pelée, Montagne (Martinique) }
\end{array}
$$


3. Réserve faunique des Laurentides (Québec)

plutôt que :

Laurentides, Réserve faunique des (Québec)

\section{Chronologie}

Cette catégorie comprend les concepts généraux non datés qui désignent de larges périodes historiques, des courants de civilisation, des ères géologiques. Elle comprend aussi des années, des décennies, des demi-siècles, des siècles et des millénaires, ainsi que certaines périodes historiques très précises. Les descripteurs chronologiques représentent $0,7 \%$ de l'ensemble des descripteurs du thésaurus.

$\begin{array}{ll}\text { Exemples: } & \text { 1. } 1900-1949 \\ & \text { 2. } 1968 \\ & \text { 3. } 18^{\mathrm{e}} \text { siècle } \\ & \text { 4. } 11 \text { septembre } 2001 \\ & 5 \cdot 2^{\mathrm{e}} \text { millénaire } \\ \text { 6. Moyen Âge } \\ \text { 7. Tertiaire (Ère géologique) }\end{array}$

\section{Descripteurs de forme}

Cette catégorie de descripteurs comprend les termes qui décrivent le type de publication, la forme ou le genre du document. Ces descripteurs, qui représentent $0,2 \%$ du lexique de RASUQAM, correspondent aux subdivisions de forme des vedettes-matière.

$$
\begin{array}{ll}
\text { Exemples : } & \text { 1. Biographie (Descripteur de } \\
\text { forme) } & \\
\text { 2. Guide touristique (Descripteur } \\
\text { de forme) } \\
\text { 3. Ressource Internet (Descripteur } \\
\text { de forme) }
\end{array}
$$

\section{Descripteurs non contrôlés}

Cette catégorie comprend les termes pour lesquels aucun travail d'autorité n'est effectué. Ils ne sont rattachés à aucun réseau sémantique et rarement possèdent-ils des formes équivalentes. Ce sont principalement des noms de tests, de collections ou d'expositions. Ils comptent pour $0,9 \%$ de l'ensemble de RASUQAM.

$$
\begin{array}{ll}
\text { Exemples: } & \text { 1. Arrêt Kosmopoulos } \\
& \text { 2. Crèches de trois continents } \\
\text { (Exposition) } & \text { 3. Health problems checklist (Test) }
\end{array}
$$

La présence de ces descripteurs non contrôlés dans le thésaurus résulte de l'obligation qui était faite, dans SIGIRD, de créer une notice d'autorité par descripteur. N'ayant pu, au moment de passer au système Manitou, les transférer dans la zone 653 qui était réservée aux seuls unitermes, la décision fut prise de leur attribuer une zone locale et de continuer la création de notices d'autorité minimales.

\section{RASUQAM et format MARC}

Depuis juin 2002, date d'implantation du module de catalogage de MANITOU, les descripteurs de RASUQAM sont créés et utilisés conformément au format MARC 21. Tous les descripteurs créés avant cette date ont fait l'objet d'une opération de conversion de données ${ }^{24}$ visant à les rendre compatibles avec le MARC 21.

La présente section donne un aperçu de l'utilisation de certaines zones et sous-zones du format MARC dans RASUQAM.

\section{Zones et sous-zones MARC}

Dans la notice d'autorité, en plus des zones réservées à la forme acceptée, aux formes rejetées, aux relations sémantiques et aux sources consultées, RASUQAM utilise quelques zones répondant à ses besoins particuliers. C'est le cas des zones 019 (caractéristiques locales), 072 (domaine d'emploi), $\mathrm{X}_{55}$ (descripteurs de forme), $\mathrm{X}_{59}$ (descripteurs non contrôlés), 690 (définition), ainsi que 750 (liaison avec le RVM).

Dans la notice bibliographique, les descripteurs sont inscrits dans les zones 6XX. Les descripteurs de forme sont en 655 et les descripteurs non contrôlés en 659.

\section{Utilisation des sous-zones}

Seule la sous-zone \$a est utilisée dans les zones $\mathrm{X}_{50}, \mathrm{X}_{51}, \mathrm{X}_{55}$ et $\mathrm{X}_{59}$ de la notice d'autorité. Elle est également la seule à être utilisée dans les zones $65 \mathrm{X}$ de la notice bibliographique.

$$
\begin{array}{cl}
\text { Exemples: } & \mathrm{X}_{50} \text { \$aÉtalement urbain } \\
& \mathrm{X}_{51} \text { \$aÉtats-Unis } \\
& \mathrm{X}_{55} \text { \$aDictionnaire (Descripteur de } \\
& \text { forme) } \\
\mathrm{X}_{59} \text { \$aArrêt Kosmopoulos }
\end{array}
$$

Le contenu des sous-zones $\$ v, \$ x$, $\$ y$ et $\$ z$ (subdivisions de forme, générale, chronologique et géographique) qu'utilisent les vedettes-matière est, selon le cas, intégré dans un descripteur plus spécifique ou exprimé à l'aide de plusieurs descripteurs.

Un cas de pré-coordination conduira à la création d'un descripteur plus spécifique dans lequel

24. La conversion des titres-sujet et des collectivités-sujet au format MARC ne s'est toutefois pas toujours effectuée correctement (par exemple, on peut retrouver dans une même sous-zone l'information qui aurait dû être transférée dans deux sous-zones différentes). Les ajustements nécessaires sont apportés ponctuellement lorsque les cas problèmes sont rencontrés. 
l'information équivalant, par exemple, à une subdivision des vedettes-matière sera intégrée à la souszone $\$$ a du descripteur.

Exemple 1650 \$aForêt tropicale plutôt que : 650 \$aForêts \$zRégions tropicales

Exemple 2650 \$aSite d'enfouissement sanitaire plutôt que : 650 \$aDéchets \$xÉlimination \$xSites

Un cas de post-coordination forcera l'utilisation de descripteurs distincts dans la notice bibliographique.
Exemple 1650 \$aPersonne âgée
650 \$aTrouble de la mémoire
plutôt que :
650 \$aMémoire, Troubles de la, chez les personnes âgées
Exemple 2650 \$aÉducation des femmes 650 \$aMoyen Âge
651 \$aFrance
plutôt que :
650 \$aFemmes \$xÉducation \$zFrance \$xHistoire \$y50o- 1500 (Moyen Âge)

\section{Cas particuliers}

Les sous-zones disponibles dans le MARC ne sont pas toutes utilisées pour coder l'information relative aux collectivités-sujet et aux titres-sujet. La structure des zones MARC qui leur sont dédiées est parfois inutilement contraignante pour un thésaurus. Sans contrevenir à la structure du format MARC, RASUQAM utilise pour ces cas particuliers des descripteurs dont la forme est plus proche des utilisateurs.

\section{Collectivités-sujet}

Cette catégorie de descripteurs utilise les zones $\mathrm{X}_{10}$ (noms de collectivités) et $\mathrm{X}_{11}$ (noms de réunions). La majorité de ces descripteurs ne comportent qu'une forme principale ou une forme principale avec unité subordonnée. Les sous-zones \$a et \$b sont utilisées avec les X1o et les sous-zones \$a et \$e sont utilisées avec les X11.

Exemples : X1o \$aBiodôme de Montréal

X1o \$aQuébec (Province). \$bMinistère de la Justice

X11 \$aFestival international de jazz de Montréal

X11 \$aCycle d'Uruguay (1978-1994). \$eComité des négociations commerciales
Les sous-zones $\$ n$, $\$ d$ et $\$ c$ ne sont pas utilisées avec les noms d'organismes dans RASUQAM. L'information qui est habituellement codée dans ces souszones est plutôt inscrite dans la sous-zone \$a du descripteur.

$$
\begin{gathered}
\text { Exemple } 1 \text { X11 \$aJeux Olympiques de } \\
\text { Montréal (1976) } \\
\text { plutôt que: } \\
\text { X11 \$aJeux Olympiques \$n(21e } \\
\text { \$d1976 \$cMontréal, Québec) }
\end{gathered}
$$

Exemple 2 X11 \$aSommet des Amériques (Québec, 2001) plutôt que :

X11 \$aSommet des Amériques \$n(3e \$d2001 \$cQuébec, Québec)

\section{Titres-sujet}

Conformément au format MARC, cette catégorie de descripteurs utilise, selon le cas, les zones Xoo, X1o, $\mathrm{X}_{11}$ et $\mathrm{X}_{30}$.

Exemples: Xoo \$aVian, Boris, \$d1920-1949. stL'écume des jours

X1o \$aQuébec (Province). \$tLoi sur les normes du travail

X11 \$aConcile du Vatican (2e : 19621965). \$tDeclaratio de libertate religiosa

X30 \$aManuscrits de la mer Morte

Certaines sous-zones ne sont pas utilisées pour les titres-sujet dans RASUQAM. C'est le cas, en particulier, des sous-zones $\$ n$ et $\$ r$ pour les zones Xoo, ainsi que $\$ n$ et $\$ d$ des $X_{30}$. L'information habituellement codée dans ces autres sous-zones sera inscrite dans la sous-zone $\$$ a (pour un titre uniforme) ou $\$ \mathrm{t} \mathrm{du}$ descripteur.

Exemple

X10 \$aBeethoven, Ludwig van, \$d1770-1827. \$tSymphonie no 9 en ré mineur, op. 125

plutôt que :

X1o \$aBeethoven, Ludwig van, \$d1770-1827. \$tSymphonies, \$nno 9, op. 125, \$rré mineur.

$$
\begin{aligned}
\text { Exemple } 2 & \text { X30 \$aTraité d'Utrecht (1713) } \\
& \text { plutôt que : } \\
& \text { X30 \$aTraité d'Utrecht \$d(1713) }
\end{aligned}
$$

\section{Descripteurs vs Vedettes-matière}

Le Service des bibliothèques de I'UQAM, peu après la création de l'Université, a fait le choix de l'automatisation et de la consultation en ligne de son catalogue. Déjà habitué, avec les unitermes, au vocabulaire libre et à la post-coordination, il a choisi 
de demeurer dans la tradition des bases de données en optant pour les descripteurs.

Les descripteurs ne comportent ni inversion ni subdivision. Les sujets complexes sont exprimés à l'aide de plusieurs descripteurs indépendants plutôt qu'en les pré-coordonnant au moyen d'une syntaxe contraignante ${ }^{25}$. Le repérage, par post-coordination, peut ainsi se faire de façon plus intuitive, sans que l'usager n'ait à connaître de règles précises. Tout en évitant la création de vedettes composées ou complexe, cette approche assure une meilleure croissance de la banque de descripteurs ${ }^{26}$ tout en facilitant le développement et la cohésion des réseaux sémantiques.

De leur côté, les vedettes-matière, créées à l'origine pour les catalogues sur fiches consultables sur place, sont un langage d'indexation pré-coordonné. Elles utilisent des règles de syntaxe très précises que chaque analyste doit respecter afin d'assurer la cohérence de l'indexation. La connaissance par l'usager de la structure des vedettes-matière peut contribuer au succès de sa recherche.

Tenant compte de l'utilisation généralisée des vedettes-matière et dans le souci de permettre aux utilisateurs externes d'interroger plus facilement le catalogue de l'UQAM ${ }^{27}$, deux moyens sont utilisés pour favoriser l'accessibilité et éviter l'isolement: la compatibilité et l'interconnexion avec le RVM.

\section{Compatibilité avec le RVM}

En autant que cela ne contrevienne pas à l'usage courant et à l'esprit du langage naturel, le choix de la forme acceptée des descripteurs se rapproche le plus possible de celle de la vedette-matière correspondante dans le RVM. Ainsi, un grand nombre des descripteurs RASUQAM se présentent exactement sous la même forme que la vedette-matière correspondante, ou sous une forme qui ne diffère que par le nombre grammatical.

$$
\begin{aligned}
& \text { Exemples : 1. Économie sociale } \\
& \text { 2. Encéphalopathie spongiforme } \\
& \text { bovine } \\
& \text { 3. Industrie chimique (RVM : } \\
& \text { Industries chimiques) } \\
& \text { 4. Littérature française }
\end{aligned}
$$

25. Cela est particulièrement vrai lorsque l'énoncé comporte plusieurs concepts identifiables.

Exemple: Personne âgée + Prévention des accidents + Québec (Province)

plutôt que :

Personnes âgées-Accidents-Québec (Province)-

Prévention

26. À titre d'exemple, «Ex-URSS» n'est présent qu'une seule fois dans RASUQAM, alors que nous pouvons trouver quelques centaines de sujets construits avec ce terme dans les catalogues utilisant les vedettes-matière. L'expression «Personne âgée» fait partie de neuf descripteurs alors qu'elle apparaît dans plusieurs centaines de vedettes construites.

27. Via la norme $Z_{39.50}$ ou en accédant directement au catalogue.
5. Pompe à béton (RVM : Pompes à béton)

6. Travaux publics

7. Violence au cinéma

Une forme différente est évidemment adoptée dans le cas de vedettes subdivisées ou inversées, puisque les pratiques de subdivision et d'inversion n'existent pas dans RASUQAM.

Exemple 1: Droit de la famille

$$
\text { RVM: Famille-Droit }
$$

Exemple 2: Harry Potter (Personnage fictif)

RVM: Potter, Harry (Personnage fictif)

Exemple 3: Lutte contre les mauvaises herbes

RVM: Mauvaises herbes, Lutte contre les

Exemple 4: Philosophie de l'histoire RVM: Histoire-Philosophie

Pour des raisons d'usage, de logique ou simplement pour faciliter l'interrogation du catalogue, la forme du descripteur RASUQAM sera aussi, dans d'autres cas, différente de celle de la vedette-matière correspondante.

Exemple 1: Alimentation électrique

RVM: Alimentation en énergie électrique

Exemple 2: Civilisation gallo-romaine

RVM: Gaule-Civilisation-58 av. J.-C.-511

Exemple 3 : Clientèle cible

RVM: Cibles (Marketing)

Exemple 4: $\mathrm{E}=\mathrm{mc2}$

RVM: Relativité (Physique)

Exemple 5 : Étalement urbain

RVM : Sol, Utilisation urbaine du

Villes-Croissance

Exemple 6: Maladie psychosomatique

RVM: Médecine psychosomatique

Exemple 7 : Pays de l'OCDE

RVM : Pays de l'Organisation de coopération et de dévelop-

pement économique

\section{Liaison avec le RVM}

La correspondance avec les vedettes-matière et le RVM est établie lors de la création ou de la révision d'un descripteur. Lorsqu'une ou plusieurs vedettesmatière équivalentes sont identifiées, ces dernières sont inscrites dans la zone de liaison ${ }^{28}$ de la fiche d'autorité du descripteur.

Cette équivalence inclut tous les cas de figure mentionnés plus haut. Elle peut donc être exacte,

28. Seule la zone 750 est utilisée à cette fin, peu importe qu'il s'agisse d'un nom commun ou de tout autre type de descripteur. 
légèrement différente ou très différente. Même si une vedette équivalente est identifiée pour la majorité des descripteurs de RASUQAM, il n'en demeure pas moins qu'environ $20 \%$ des descripteurs sont créés sans liaison avec le RVM. L'explication de cet état de fait tient probablement à la profondeur de l'analyse et à la plus grande spécificité de l'expression des sujets. La nature des collections à traiter ainsi que la rapidité de traitement de la documentation récente peuvent également être en cause.

Voici quelques exemples de descripteurs de RASUQAM qui ne possédaient pas d'équivalent dans le RVM au moment de la rédaction de cet article:

$\begin{array}{ll}\text { Exemples : } & \text { 1. Algorithme stochastique } \\ \text { 2. Employé permanent } \\ \text { 3. Grand Maghreb } \\ \text { 4. Internet des objets } \\ \text { 5. Justice participative } \\ \text { 6. Méridien de Paris } \\ \text { 7. Robot de loisir } \\ \text { 8. Solvant chloré }\end{array}$

\section{Conclusion}

Le langage conceptuel des descripteurs, utilisé depuis 1994 pour l'indexation des collections dans les bibliothèques de l'UQAM, s'inscrit dans le prolongement de la philosophie adoptée dès les débuts de BADADUQ: langage post-coordonné, ouvert et facile d'utilisation. Innovateur dans le milieu universitaire, on retrouve dans RASUQAM les termes normalisés, l'organisation conceptuelle ainsi que les relations sémantiques (équivalences, hiérarchies et associations) qui caractérisent les véritables thésaurus.

Développé au rythme de l'indexation, RASUQAM a eu un impact positif sur la productivité des employés, laquelle a connu une hausse de $21 \%$. Maîtres d'œuvre de leur propre vocabulaire, les indexeurs de l'UQAM peuvent intégrer rapidement au thésaurus les néologismes et effectuer sans attendre les mises à jour nécessaires.

Les quelque 40000 descripteurs qui composent aujourd'hui RASUQAM, alliés à environ 225000 notices bibliographiques indexées, forment une masse critique capable de fournir des réponses pertinentes et abondantes aux requêtes des usagers. Fort de ses 80000 relations, le réseau sémantique robuste et en évolution constante de RASUQAM facilite la navigation et le raffinement des stratégies de recherche. Il est aussi d'une aide considérable au moment de l'identification des concepts à indexer.

Maintenant qu'Internet a fait passer dans les mœurs la recherche en ligne et que les usagers, de plus en plus habitués aux bases de données, interrogent volontiers à distance, l'UQAM dispose d'un thésaurus encyclopédique capable d’offrir un mode de repérage par sujet efficace, convivial et compatible avec l'autonomie et le langage de ses utilisateurs. RASUQAM est ainsi en voie de réaliser le rapprochement avec le langage courant recherché par d'autres: "What we fail to provide is seamlessness, simplicity, and common language searching. For the past 10 years online searching has become simpler and more effective everywhere, except in library catalogs ${ }^{29}$. »

La reconnaissance de RASUQAM par la Library of Congress permet aux autres institutions de conserver et d'exploiter sans problèmes les descripteurs trouvés dans les notices repêchées à l'UQAM. Celles qui le font déjà permettent à leurs usagers de bénéficier de points d'accès supplémentaires. Ainsi, un système singulier, celui des descripteurs, dont l'approche est différente de celle des vedettes-matière, peut-il contribuer à une meilleure exploitation des fonds documentaires de toutes les bibliothèques.

RASUQAM est le fruit du travail acharné d'une équipe d'indexeurs polyvalents, dévoués expérimentés et compétents. C'est grâce à l'effort de chacun d'eux que ce projet, qui ne manque pas d'audace, a vu le jour et qu'il est mené à bien depuis plus de 11 ans. $(-$

\section{Note de l'auteur}

Il n'est possible, pour le moment, d'interroger le thésaurus RASUQAM qu'en passant par le catalogue $B A D A D U Q$ des bibliothèques de l'UQAM. Le lecteur intéressé est invité à consulter la page Web du Service de l'analyse documentaire où il trouvera l'information nécessaire pour accéder au thésaurus RASUQAM : $<$ http://www.bibliotheques.uqam.ca/bibliotheques/ serv_techniques/analyse/index.html>.

29. University of California Libraries. Bibliographic Services Task Force. Rethinking How We Provide Bibliographic Services for the University of California. Final report, décembre 2005, p. 11. URL: <http://libraries.universityofcalifornia.edu/ sopag/BSTF/Final.pdf>. 